\title{
Postinfectious Olfactory Dysfunction: Oral Steroids and Olfactory Training versus Olfactory Training Alone: Is There any Benefit from Steroids?
}

\author{
Sotiria Genetzaki Evangelia Tsakiropoulou Vasilios Nikolaidis \\ Konstantinos Markou Iordanis Konstantinidis \\ 2nd Academic ORL Department, Papageorgiou Hospital, Aristotle University, Thessaloniki, Greece
}

\section{Keywords}

Anosmia $\cdot$ Olfactory training $\cdot$ Postinfectious olfactory

dysfunction · Steroids $\cdot$ Smell

\begin{abstract}
Introduction: There are limited treatment options for postinfectious olfactory dysfunction (PIOD). Olfactory training has recently been used in clinical practice, but no medical treatment is widely accepted. Although there is weak evidence for their value, some physicians use oral corticosteroids as first-line treatment. The aim of this study was to compare combined oral methylprednisolone and olfactory training with olfactory training alone in the management of PIOD. Methods: This prospective cohort study included 131 patients with PIOD over a 2-year period before the COVID-19 pandemic. Seventy-eight patients who were treated with oral methylprednisolone and olfactory training (group A) were compared with 53 patients who were treated with olfactory training only (group B). Olfactory function was evaluated with "Sniffin' Sticks" at baseline and 2, 8, and 16 weeks after initial assessment. Patients who improved after steroid treatment underwent magnetic resonance imaging of the paranasal sinuses, skin prick tests, lung spirometry, and sputum eosinophil assessment. Results: Oral steroids improved $19.23 \%$ of patients ( $n=15)$ of group A. History, clinical evaluation, imaging, and laboratory tests identified an inflamma-
\end{abstract}

karger@karger.com www.karger.com/orl

(C) 2021 S. Karger AG, Basel

Karger ${ }^{\prime \prime}=$ tory background in half of them $(n=8)$. The remaining 7 had no findings of nasal inflammation, and all had a short history of olfactory dysfunction. Both groups significantly improved in olfactory testing results at the end of the olfactory training scheme without significant difference between them. Conclusions: The percentage of improved patients after oral methylprednisolone was relatively low to suggest it as firstline treatment. Half of the improved patients had an underlying upper airway inflammatory condition not related to the infection that caused the acute loss of olfactory function.

(c) 2021 S. Karger AG, Basel

\section{Introduction}

It is estimated that approximately $15 \%$ of the general population suffers from olfactory impairment [1]. This condition has a negative effect on a person's quality of life and can lead to depression, social isolation, disturbed eating habits, and exposure to environmental dangers [2]. The most common causes of olfactory loss are chronic sinusitis, upper respiratory tract infection (URTI), head trauma, and idiopathic disease [2].

The present study focuses on postinfectious olfactory dysfunction (PIOD), which is often of viral origin. Specifically, our study includes only patients with the socalled "classic" PIOD in contrast with the COVID-19-re- 
lated PIOD. The collected data reflect a time period before the pandemic; however, as there are similarities with the olfactory loss caused by SARS-CoV-2, our results can be used for treatment plan strategies against it. Typically, after an acute respiratory tract infection, such as a common cold or influenza, there is a persistent loss of smell despite resolution of the sinonasal symptoms. Reden et al. [3] reported that approximately one-third of these patients may experience spontaneous resolution of their symptoms during the next 2 years; however, their data did not include patients with short-lasting olfactory loss. There are limited treatment options for PIOD, and none of them are universally accepted. The literature presents a growing body of evidence that olfactory training with repeated exposure to odours is a promising modality. It is a low-cost and safe method that relies on the unique plasticity of the olfactory system. Medical therapy often includes topical or systemic corticosteroids, but although they remain the most commonly prescribed drugs for olfactory loss, their benefit for non-sinonasal disease cases remains unclear. For PIOD, the rationale is that they mainly reduce the subclinical inflammation that persists after URTI. Previous studies have reported relatively high percentages (32$55 \%$ ) of improved PIOD patients after a short course of oral steroids [4, 5]. Fleiner et al. [6] concluded that augmented olfactory training with a topical nasal steroid was more effective than olfactory training alone in patients of mixed aetiology; however, this fact was not evident in the PIOD subgroup. Furthermore, Nguyen and Patel [7] found that olfactory ability significantly improved with a combination of nasal budesonide and olfactory training. The aim of this study was to analyse the characteristics of the subset of "classic" PIOD patients who improved after a course of oral steroids and to assess whether combined therapy with corticosteroids and olfactory training is more effective than olfactory training only.

\section{Materials and Methods}

\section{Patients}

This study included 131 patients (89 females and 42 males) who presented or were referred to our Smell and Taste Clinic with "classic" PIOD over a period of 2 years. The age range was from 22 to 79 years, and the mean age was $51.45 \pm 7.2$ years.

Acute olfactory impairment following a history of URTI was the prerequisite for participation in the study. Patients had to be able to recall when the episode of URTI occurred within a range of 3 weeks; otherwise, the diagnosis was reconsidered. The exclusion criteria were pregnancy, diagnosis of acute/chronic rhinosinusitis with or without polyps, neurodegenerative diseases, history of head trauma, congenital and idiopathic olfactory dysfunction, chronic or recent short-term oral steroid use, and history of nasal and/or neurosurgical procedures. The study was conducted in accordance with the Declaration of Helsinki for Medical Research Involving Human Subjects and received the approval of the Ethics Review Committee of Aristotle University of Thessaloniki (9201/22-7-2017). All participants provided written informed consent prior to their participation after detailed explanation of the study design.

\section{Clinical Evaluation}

During the first visit, a fully detailed medical history concerning diagnosed conditions (such as rhinitis, chronic rhinosinusitis, and asthma) or symptoms suggestive of upper airway inflammatory disorders (including nasal obstruction, rhinorrhea, sneezing, and postnasal drip) was obtained. Participants were asked to report any medications they used. Factors such as duration of olfactory loss and presence of parosmia or phantosmia were also assessed. Specifically, patients had to indicate whether they experienced distorted or phantom odours (yes vs. no). Every patient underwent a thorough clinical ear, nose, and throat examination and nasal endoscopy with special attention to the visibility and patency of the olfactory cleft. The presence of polyps or oedema in the olfactory cleft was recorded, along with general findings suggesting nasal inflammation, such as secretions, turbinate hypertrophy, and mucosal irritation.

\section{Olfactory Testing}

Clinical evaluation of olfactory function was performed using "Sniffin' Sticks" (Burghart GmbH, Wedel, Germany) testing and the Greek verbal version [8]. This olfactory test battery has 3 subsets: the phenyl ethyl alcohol odour threshold (T), odour discrimination (D), and odour identification (I). Each test has a maximum score of 16 points. The total sum obtained (maximum 48 points) is reported as the TDI score. With the use of Sniffin' Sticks, olfactory function is classified as normosmia (TDI > 30.5), hyposmia (TDI: 16.5-30.5), and functional anosmia (TDI < 16.5) [9]. Previous studies have determined that an increase of 5.5 or more points of the TDI score can be regarded as clinical improvement of olfactory function [10]. In addition, subjective assessment of olfactory function was performed by means of a visual analogue scale with a score ranging from 0 to 100 in which 0 represents total olfactory loss and 100 represents perfect olfactory function.

\section{Procedure}

The oral steroid course and the olfactory training procedures were explained in detail to all participants. Patients with contraindications for oral steroids (including uncontrolled diabetes, osteoporosis, and high blood pressure) had 2 options: either to follow the olfactory training scheme or to wait for spontaneous recovery. The rest of the patients had to decide to follow either the combined scheme (steroids + olfactory training) or olfactory training alone or wait for spontaneous recovery.

Only 4 patients chose not to follow any treatment and wait for spontaneous recovery, so they were excluded from the study. Thus, 2 study groups were analysed: (1) patients who received oral steroids and followed the olfactory training scheme (group A), and (2) patients who only followed the olfactory training scheme (group B).

All patients were assessed at 2, 8, and 16 weeks from baseline assessment. Olfactory function was examined during each visit 
with subjective ratings and psychophysical olfactory testing. The standard clinical practise in smell and taste or general ear, nose, and throat clinics when dealing with PIOD patients is the assessment with nasal endoscopy and olfactory testing with psychophysics without imaging or other special laboratory studies [2].

Based on previous experience of clinicians using oral steroids as diagnostic tool where responders are considered as patients having a conductive component in their olfactory loss, we decided to firstly prescribe oral steroids and secondly to offer a package of laboratory tests only to oral steroid responders. Thus, patients who improved after oral methylprednisolone were assessed with magnetic resonance imaging (MRI) of the paranasal sinuses for detection of undiagnosed sinus disease, skin prick tests (SPTs), lung spirometry, and sputum eosinophil count, regardless of whether a diagnosis of allergic rhinitis or asthma had already been established.

No staging system was used for imaging studies in the present cohort as MRI findings are characterised as positive or negative for the presence of chronic rhinosinusitis. Specifically, an MRI was considered positive when thickened mucosa was found in at least 1 sinus and/or middle meatus, with the exception of maxillary retention cysts or history of odontogenic maxillary sinusitis.

Spirometry was considered to be suggestive of asthma in undiagnosed patients when the forced expiratory volume in the first second predicted value was $<80 \%$ [11]. In diagnosed asthma patients, a forced expiratory volume in the first second $<80 \%$ was considered moderate disease and $\leq 60 \%$ as severe disease. A sputum eosinophil count $\geq 3 \%$ was considered indicative of eosinophilic upper airway inflammation [12]. Asthma patients were recorded according to the Global Initiative of Asthma 2020 (GINA 2020) as slightly persistent, moderately persistent, and severely persistent asthma [13]. Asthmatic patients with an eosinophil count $\geq 3 \%$ were considered having eosinophilic asthma. Allergic rhinitis patients were divided according to the Allergic Rhinitis and its Impact on Asthma (ARIA) classification regarding the severity of symptoms as patients having mild and moderate/severe allergic rhinitis [14].

\section{Oral Steroid Course}

Patients were administered a course of oral methylprednisolone for 14 days. The initial dose was $40 \mathrm{mg}$ orally, and the dosage was decreased by $5 \mathrm{mg}$ every 2 days. Each patient was given detailed and printed instructions about the treatment schedule and how to follow it. Additionally, all participants in the combined scheme were informed about methylprednisolone and its possible side effects. Patients who discontinued the treatment before completing 2 weeks of administration or did not adhere to the schedule were excluded from the study.

\section{Olfactory Training}

Olfactory training was based on daily exposure to specific odorants selected from the 4 primary odour categories (fruity, flowery, spicy, and resinous) as described by Henning's Odor Prism [15]. In particular, all patients were exposed for $5 \mathrm{~min}$ twice a day to 4 odours: citronella (lemon), phenyl ethyl alcohol (rose), eugenol (cloves), and eucalyptol (eucalyptus), as used in previous studies $[16,17]$. During each session, odours were delivered to the patient's nose for $10 \mathrm{~s}$ and then rotated with an interval of $10 \mathrm{~s}$ between them. Patients were asked to keep a diary where they noted every session of training day by day. These notes were assessed regarding compli-

Oral Steroids and Olfactory Training

versus Olfactory Training Alone ance with training at every follow-up assessment at 2, 8, and 16 weeks. Patients who did not comply with the training scheme for at least 7 days were excluded from the study. Patients had to follow this olfactory training scheme over a period of 16 weeks.

\section{Statistical Analysis}

The data were analysed using SPSS 20.0 (SPSS Inc., Chicago, IL, USA). Demographic and clinical data are reported as mean \pm standard deviation or percentage (\%). The unpaired $t$ test and Pearson's correlation coefficient were used to compare the 2 study groups. The Bonferroni adjustment was used for post hoc analysis. The alpha level was set at $p=0.05$.

\section{Results}

The present study included 131 out of 142 examined patients over a period of 2 years. Eleven patients were excluded as 4 patients chose to wait for spontaneous recovery and 7 patients did not correctly follow the steroid treatment and/or the olfactory training scheme (7 out of 138; treatment discontinuation rate: 5.07\%). Group A (steroid course + olfactory training) included 78 patients, while group B (olfactory training only) included 53 patients. Both groups had a similar distribution of anosmia and hyposmia incidence (group A: 41 anosmics [52.5\%] and 37 hyposmics [47.5\%], group B: 30 anosmics [56.6\%] and 23 hyposmics [43.4\%]). Parosmia was reported by 11 patients in group A and 6 patients in group B (14.1 and $11.3 \%$, respectively). None of the included patients reported phantosmia.

\section{Steroid Treatment}

No side effects were reported after treatment with methylprednisolone. The data showed that only $19.23 \%$ ( $n=15$ of 78 patients) showed significant improvement of TDI scores after 2 weeks of the steroid course. During the second appointment, all patients who had improved underwent MRI of the paranasal sinuses, SPTs, lung spirometry, and assessment of sputum eosinophils. Eight of these 15 patients had some underlying inflammatory airway disease. Specifically, 4 patients had findings of paranasal sinus and/or olfactory cleft inflammation, 4 patients had a positive SPT and nasal symptoms suggestive of allergic rhinitis, and 3 patients had positive sputum eosinophilia. Interestingly, only 4 of the 8 patients had a previously diagnosed inflammatory airway disorder (2 with allergic rhinitis and 2 with asthma). None of the 4 patients with chronic rhinosinusitis findings on MRI had a previous diagnosis of sinusitis. All the above-mentioned findings in the improved patients are presented in Table 1. 


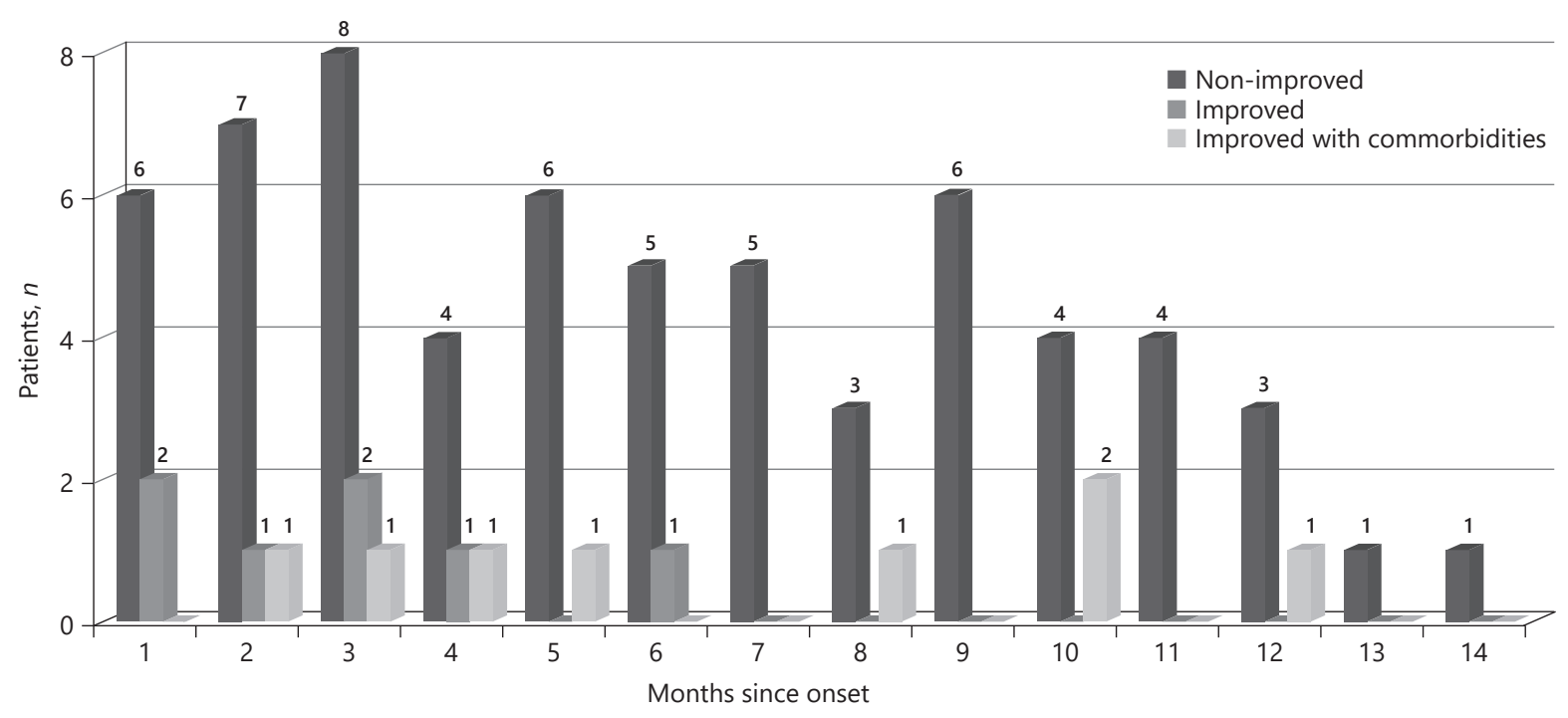

Fig. 1. Distribution of improved and non-improved patients of the group who received combined treatment after 2 weeks of oral steroid treatment in relation to their history of disease.

Table 1. Improved patients with upper airway inflammatory background $n=8 / 78$

\begin{tabular}{|c|c|c|c|c|c|c|c|}
\hline \multicolumn{4}{|c|}{ Baseline assessment } & \multicolumn{4}{|c|}{ After oral steroid course } \\
\hline patients & history & endoscopy & reported symptoms & MRI & SPT & $\begin{array}{l}\text { lung } \\
\text { spirometry }\end{array}$ & $\begin{array}{l}\text { sputum } \\
\text { eosinophil }\end{array}$ \\
\hline 1 & - & + & - & + & - & - & - \\
\hline 2 & - & - & Nasal obstruction & + & - & - & - \\
\hline 3 & Asthma (moderate) & - & Postnasal drip, cough & + & - & + & + \\
\hline 4 & AR (mild/moderate) & - & - & - & + & - & - \\
\hline 5 & AR (severe) & - & Rhinorrhea, nasal obstruction & - & + & - & - \\
\hline 6 & Asthma (moderate) & - & Nasal obstruction & - & + & + & + \\
\hline 7 & - & - & Nasal obstruction & + & - & - & + \\
\hline 8 & - & - & Sneezing, rhinorrhea & - & + & - & - \\
\hline
\end{tabular}

History and laboratory findings of patients who improved after oral methylprednisolone with evidence of upper airway inflammatory background. At baseline assessment, previously diagnosed conditions are presented in column history. Positive endoscopy corresponds to findings of inflammation as described in the Mat-methods section, and reported symptoms correspond to suggestive symptoms of upper airway inflammatory conditions within the last 6 months. The severity of asthma and allergic rhinitis was classified according to GINA13 and ARIA14 guidelines, respectively. After the oral steroid course, positive MRI corresponds to thickened mucosa in at least 1 sinus and/or middle meatus; positive SPT corresponds to positive skin reaction to at least 1 allergen; positive lung spirometry corresponds to an $\mathrm{FEV}_{1}$ predicted value $<80 \%$; and positive sputum eosinophil corresponds to a eosinophil count $\geq 3 \%$. SPT, skin prick test; MRI, magnetic resonance imaging; $\mathrm{FEV}_{1}$, forced expiratory volume in the first second.

Subjective ratings after oral steroid treatment showed a higher percentage of improved patients $(n=18 ; 23.07 \%)$. However, 3 patients were found to have no significant change when tested with the Sniffin' Sticks. Response to steroid treatment was not related to gender $(p=0.70)$ or presence of parosmia before therapy $(p=0.49)$. The dura- tion of disease in group A was not related to treatment response $(p=0.23)$. However, when comparing the mean duration of disease between improved patients with an inflammatory background and those who improved without any evidence of nasal inflammation, the latter subgroup had a significantly shorter history of disease (mean: 
Fig. 2. TDI scores during the study period of group A steroid responders, group A nonsteroid responders, and group B. Asterisk indicates significantly higher TDI results of group A steroid responders at 2 weeks assessment than with TDI results of group A non-steroid responders and group B.

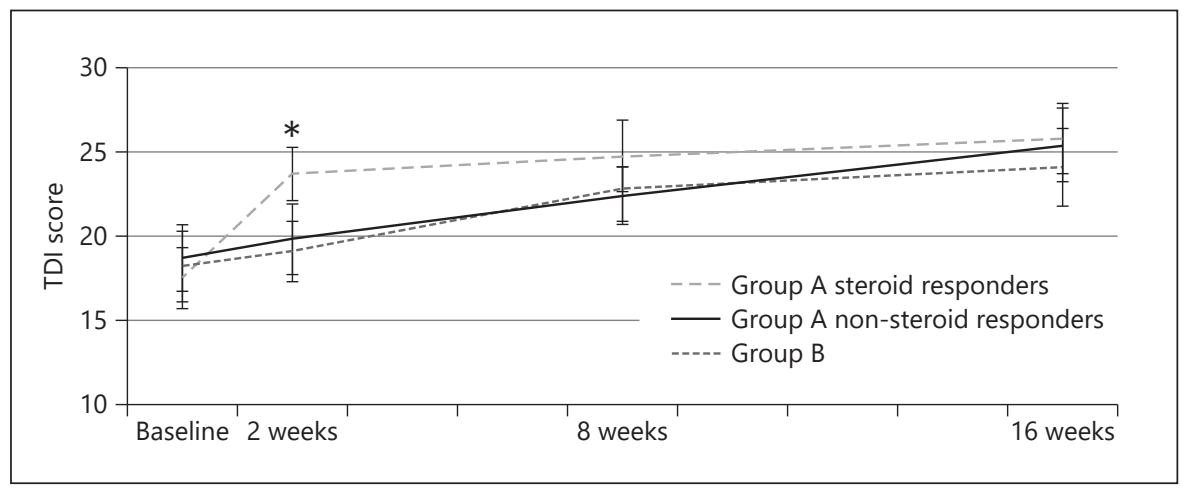

$6.85 \pm 1.8$ months vs. $2.85 \pm 1.2$ months; $p=0.003$ ). Figure 1 presents the distribution of improved and non-improved patients in group A after 2 weeks of steroid treatment in relation to their history of disease. In the nonimproved 63 patients of group A, only 3 patients had a history of diagnosed airway inflammation ( 1 of well-controlled mild asthma and 2 of idiopathic rhinitis) and 1 patient had symptoms suggestive of rhinitis (rhinorrhea).

\section{Olfactory Training Treatment}

In general, olfactory training was beneficial for both groups. TDI scores increased from a mean of $18.5 \pm 2$ to $25.5 \pm 2.2$ in group A and $18.2 \pm 1.6$ to $24.1 \pm 1.8$ in group $\mathrm{B}$ at the 16-week follow-up assessment. There was no significant difference between the mean increases.

Specifically, the subgroup of patients in group A who improved after steroid treatment showed significantly better olfactory test results in 2 weeks $(p=0.31)$. However, this was not the case at the 8- and 16-week follow-up assessments (Fig. 2). The percentage of improved patients was similar in both groups at the last follow-up assessment, at $55 \%$ for group A and $51 \%$ for group B (Fig. 3). No correlation was found within the 131 study participants (groups A and B) between gender $(r=0.16, p=0.25)$, age $(r=-0.11, p=0.09)$, duration of disease $(r=-0.08, p=$ 0.32 ), and olfactory test results at the end of the study.

\section{Discussion}

The aim of this prospective study was to investigate the effectiveness of a short course of oral methylprednisolone combined with olfactory training compared to olfactory training alone in the treatment of "classic" PIOD. While proven that steroids are helpful for sinonasal olfactory dysfunction, their use for olfactory dysfunction of other aetiologies is under question.

Oral Steroids and Olfactory Training versus Olfactory Training Alone

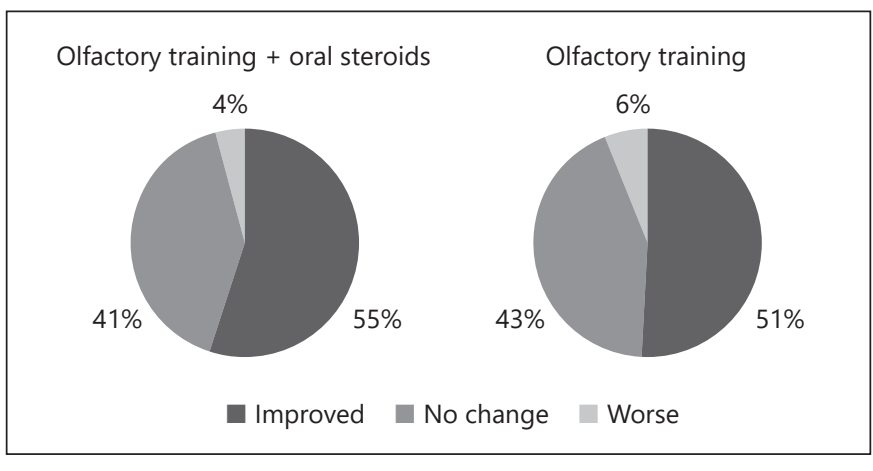

Fig. 3. Comparison of percentages of improved patients between the 2 treatment options: olfactory training and oral steroids versus olfactory training.

This study had three major outcomes:

1. Oral methylprednisolone improved a relatively low proportion of patients with post-URTI olfactory loss.

2. Imaging, laboratory tests, and detailed history revealed an underlying inflammatory airway disorder in half of the improved patients.

3. A short course of oral steroids did not modify the prognosis of the disease, as seen in the comparison between patients who received oral steroids and patients who did not.

The percentage of patients who improved with steroids is considered too low to recommend them as firstline treatment. There is a lack of the literature regarding oral steroids and post-URTI olfactory loss. Two recent reviews revealed only 7 studies on systemic steroid use in non-chronic rhinosinusitis olfactory loss patients with overall weak evidence to support their use $[18,19]$. This is because these studies are mainly at a low level of evidence ( 1 of level 3 and 6 of level 4 ) and are retrospective or non-controlled, so they are susceptible to risk of random error. Specifically, a study by Ikeda et al. [20] report- 
ed no improvement in post-URTI patients after oral steroid treatment. Heilmann et al. [21], Stenner et al. [22], and Schriever et al. [23] reported higher percentages of improved patients than the present study, with the latter 2 studies reporting 30 and $29.6 \%$ in post-URTI patients, respectively [21-23]. However, these studies were of mixed aetiology, assessed a relatively small number of post-URTI patients, and had results that were difficult to interpret. Two larger studies by Kim et al. [24] and Seo et al. [4] reported higher recovery percentages after oral steroid treatment $[4,24]$. Specifically, Kim et al. [24] reported that 178 post-URTI patients had a $55 \%$ recovery rate after oral steroids, but this was self-reported by the patients, and the smell test differences were marginal [24]. In their study, Seo et al. [4] reported a 32\% recovery rate when testing the odour threshold, but the recovery rate was much smaller for odour identification (14\%) [4]. In general, the higher percentages of improvement in other studies may possibly be attributed to the small size of cohorts and the timing of their follow-ups, where spontaneous recovery can blur the results and when patients with undetected sinonasal disease are included.

In addition, studies exploring oral steroids as treatment of PIOD patients did not clarify if 1 formulation or dose is better than others as comparable studies of different oral steroids do not exist. The option of local steroid administration by means of nasal sprays seems not really useful as they do not reach the olfactory cleft [25]. However, the use of special cannulas on nasal spray devices or nasal drops with specific head positioning provides a better application of steroids into the olfactory cleft $[26,27]$.

Schriever et al. [23] discussed the results of an idiopathic olfactory loss patient group and speculated that a percentage of this group may have inflammation in the olfactory cleft that cannot be observed macroscopically. Based on that, they suggested that patients with undetected sinonasal olfactory dysfunction are expected to respond better to treatment with systemic steroids than those with real idiopathic olfactory dysfunction. This is in agreement with our findings as an inflammatory background was found in half of those who responded to oral steroid treatment.

The unified airway theory should make rhinologists focus not only on chronic nasal inflammation but also on pulmonary-associated inflammatory disorders like asthma. The link between chronic rhinosinusitis and asthma is well-established, and it seems that olfactory dysfunction in the presence of asthma indicates similar inflammatory mechanisms in both the upper and lower airways.
Kanemitsu et al. [28] showed that sputum eosinophilia in asthmatic chronic rhinosinusitis patients was associated with olfactory dysfunction, which suggests that this impairment is a potential indicator of Th2-driven inflammation of the lower airways. In addition, although there were no such patients in the present study, it seems that a diagnosis of bronchiectasis may also be associated with chronic rhinosinusitis and olfactory loss [29]. Thus, as chronic rhinosinusitis in some patients could be subclinical, a positive history of inflammatory lower airway disease may be useful to predict a positive response to oral steroids.

Although the benefit of steroids for post-URTI olfactory loss is not entirely clear, physicians still commonly use them as first-line treatment. Some authors suggest that chronic post-URTI olfactory loss is correlated with persistent subclinical inflammation in the olfactory epithelium [30]. However, the usual clinical evaluation of such patients cannot rule out other causes of inflammatory changes in the olfactory epithelium as it is mainly based on history and endoscopy. A detailed history focused on certain inflammatory disorders, such as allergic rhinitis and asthma, along with laboratory studies, including imaging and SPTs, could help identify potential responders to oral steroids.

However, the cost of a full assessment regarding airway inflammatory disorders in PIOD patients would be significant. Another option could be the use of a short course of oral steroids (3-4 days) as a diagnostic tool, meaning that a positive olfactory response would confirm the presence of nasal mucosa inflammation, and a full course of steroids completing 2 weeks could follow. This approach could be value for money as it would have a significant impact on patient management by avoiding unnecessary examinations in non-responders.

The fact that both study groups had similar recovery rates at the end of the study period demonstrates that oral steroids did not modify the course of the disease. This could be explained in 2 ways: firstly, a relatively short course of steroids cannot change the course of an inflammatory mucosal process and only offer better conduction of odour in the olfactory cleft. If present, by definition, concomitant chronic rhinosinusitis is not extended and severe, and thus, it can be stated that it does not significantly affect the olfactory mucosa. Secondly, histological studies have shown that viral infections causing olfactory loss result in direct and more severe damage to the olfactory epithelium in terms of loss of cilia and changes in the shape of olfactory neurons [31]. It is uncertain if oral steroids could play a role in the regenera- 
tion process of the olfactory epithelium in PIOD patients. In vitro and in vivo animal studies have found that glucocorticoid receptors exist in the olfactory epithelium on mature ORNs and supporting cells. Nishimura et al. [32] showed that glucocorticoids could have a direct effect on $\mathrm{Na} / \mathrm{K}$-ATPase expression of rat olfactory mucosa, but they stated that this was of little benefit for the morphological regeneration of olfactory mucosa after injury. In addition, Takanosawa et al. [33] found that glucocorticoid administration induced apoptotic changes in mature olfactory neurons in the murine olfactory epithelium. These apoptotic changes in the olfactory epithelium induced by glucocorticoid administration may enhance the regeneration of the olfactory epithelium. Considering that olfactory epithelium regeneration has a turnover of about 30 days, this may indicate why steroids could be beneficial during the early recovery phase and in doubt at a later time [34]. This study's data determined that steroid responders without underlying inflammatory disease had a short history of olfactory loss. Early treatment could decrease the inflammatory process, reduce injuryassociated tissues, and accelerate olfactory neuron recovery, thereby avoiding irreversible nerve degeneration and permanent olfactory loss. However, the effects of oral steroids on olfactory mucosa need further studies to clarify the potential benefits and timing of administration.

During these days of the SARS-CoV-2 pandemic, where acute olfactory impairment is recognised as an early symptom of the disease, systemic steroids became an important therapeutic tool against COVID-19 [35]. Specifically, according to the WHO recommendations, systemic steroids are beneficial in patients with severe and critical COVID-19 infection [36]. However, in patients with non-severe disease, it may increase mortality, and thus, systemic steroids were not recommended. Considering that the majority of patients with olfactory loss have a mild form of COVID-19 disease, it seems that systemic steroids are not a first-line option as there is no evidence regarding their beneficial effect on olfactory loss.

Finally, in a recent consensus statement on the treatment of PIOD, there was some discordance within the expert panel regarding the use of oral steroids [37]. This was recommended with caution after an initial diagnostic treatment of 3-4 days, followed by 1-2 weeks in case of positive response after detailed discussion with patients about the risks (e.g., hip fractures and decompensating glaucoma) and benefits of this treatment option.

The results of this study should be interpreted considering certain limitations. Firstly, it is not a randomised study, which may introduce selection bias. Secondly, the full laboratory examination package was completed with steroid responders only. However, these examinations are not part of the everyday practise in post-URTI olfactory loss, and the focus of this study to steroid responders led to this decision. Nevertheless, this is the first study examining steroid responders in order to reveal upper airway inflammation, which provides evidence that a certain percentage of these patients have a concomitant inflammatory disorder. Further studies are needed to clarify which PIOD patients are suitable for oral steroid treatment.

\section{Conclusion}

The percentage of improved patients with postinfectious olfactory loss after a course of oral methylprednisolone was relatively low to suggest it as first-line treatment. Half of the improved patients had an underlying upper airway inflammatory condition not related to the causative infection of olfactory loss. Oral steroids did not modify the prognosis of the disease, as seen in the comparison between patients who received oral steroids and patients who did not.

\section{Statement of Ethics}

Investigations were performed according to the Guidelines for Biomedical Studies Involving Human Subjects ("Helsinki Declaration"). The protocol was approved by the Ethics Committee of Aristotle University of Thessaloniki (9201/22-7-2017), and all subjects provided written informed consent.

\section{Conflict of Interest Statement}

The authors have no conflicts of interest to declare.

\section{Funding Sources}

There is no funding arrangement related to this research.

\section{Author Contributions}

S.G.: data acquisition, analysis, and interpretation, and drafting, revision, and final approval of the manuscript. E.T.: data acquisition, and drafting, revision, and final approval of the manuscript. V.N.: revision and final approval of the manuscript. K.M.: revision and final approval of the manuscript. I.K.: study conception, and revision and final approval of the manuscript. 


\section{References}

1 Landis BN, Konnerth CG, Hummel T. A study on the frequency of olfactory dysfunction. Laryngoscope. 2004 Oct;114(10):17649.

2 Hummel T, Whitcroft KL, Andrews P, Altundag A, Cinghi C, Costanzo RM, et al. Position paper on olfactory dysfunction. Rhinol Suppl. 2017 Mar;54(26):1-30.

3 Reden J, Mueller A, Mueller C, Konstantinidis I, Frasnelli J, Landis BN, et al. Recovery of olfactory function following closed head injury or infections of the upper respiratory tract. Arch Otolaryngol Head Neck Surg. 2006 Mar; 132(3):265-9.

4 Seo BS, Lee HJ, Mo JH, Lee CH, Rhee CS, Kim JW. Treatment of postviral olfactory loss with glucocorticoids, Ginkgo biloba, and mometasone nasal spray. Arch Otolaryngol Head Neck Surg. 2009 Oct;135(10):1000-4.

5 Kim DH, Kim SW, Hwang SH, Kim BG, Kang JM, Cho JH, et al. Prognosis of olfactory dysfunction according to etiology and timing of treatment. Otolaryngol Head Neck Surg. 2017 Feb;156(2):371-7.

6 Fleiner F, Lau L, Goktas O. Active olfactory training for the treatment of smelling disorders. Ear Nose Throat J. 2012 May;91:198215.

7 Nguyen TP, Patel ZM. Budesonide irrigation with olfactory training improves outcomes compared with olfactory training alone in patients with olfactory loss. Int Forum Allergy Rhinol. 2018 Sep;8(9):977-81.

8 Konstantinidis I, Printza A, Genetzaki S, Mamali K, Kekes G, Constantinidis J. Cultural adaptation of an olfactory identification test: the Greek version of Sniffin' Sticks. Rhinology. 2008 Dec;46(4):292-6.

9 Oleszkiewicz A, Schriever VA, Croy I, Hähner A, Hummel T. Updated Sniffin' Sticks normative data based on an extended sample of 9,139 subjects. Eur Arch Otorhinolaryngol. 2019 Mar;276(3):719-28.

10 Gudziol V, Lötsch J, Hähner A, Zahnert T, Hummel T. Clinical significance of results from olfactory testing. Laryngoscope. 2006 Oct;116(10):1858-63.

11 Haynes JM. Basic spirometry testing and interpretation for the primary care provider. Can J Respir Ther. 2018 Winter;54(4).

12 Demarche SF, Schleich FN, Paulus VA, Henket MA, Van Hees TJ, Louis RE. Asthma control and sputum eosinophils: a longitudinal study in daily practice. J Allergy Clin Immunol Pract. 2017 Sep-Oct;5(5):1335-e5.
13 Global Initiative for Asthma. Global strategy for asthma management and prevention. 2020. Available from: https://ginasthma.org/ wp-content/uploads/2020/06/GINA-2020report_20_06_04-1-wms.pdf Accessed 2020 Jul 11.

14 Bousquet J, Khaltaev N, Cruz AA, Denburg J, Fokkens WJ, Togias A, et al. Allergic rhinitis and its impact on asthma (ARIA) 2008 update (in collaboration with the World Health Organization, GA(2)LEN and AllerGen). Allergy. 2008 Apr;63(Suppl 86):8-160.

15 Henning H. Der Geruch. Leipzig, Germany: Johann Ambrosius Barth; 1916. p. 365-70.

16 Hummel T, Rissom K, Reden J, Hahner A, Weidenbecher M, Huttenbrink KB. Effects of olfactory training in patients with olfactory loss. Laryngoscope. 2009 Mar;119:496-9.

17 Damm M, Pikart LK, Reimann H, Bukert S, Goktas $\mathrm{O}$, Haxel B, et al. Olfactory training is helpful in postinfectious olfactory loss: a randomized, controlled, multicenter study. Laryngoscope. 2014 Apr;124:826-31.

18 Yan CH, Overdevest JB, Patel ZM. Therapeutic use of steroids in non-chronic rhinosinusitis olfactory dysfunction: a systematic evidence-based review with recommendations. Int Forum Allergy Rhinol. 2019 Feb;9(2): 165-76.

19 Hura N, Xie DX, Choby GW, Schlosser RJ, Orlov CP, Seal SM, et al. Treatment of postviral olfactory dysfunction: an evidencebased review with recommendations. Int Forum Allergy Rhinol. 2020 Sep;10(9):1065-86.

20 Ikeda K, Sakurada T, Suzaki Y, Takasaka T. Efficacy of systemic corticosteroid treatment for anosmia with nasal and paranasal sinus disease. Rhinology. 1995 Sep;33(3):162-5.

21 Heilmann S, Huettenbrink KB, Hummel T. Local and systemic administration of corticosteroids in the treatment of olfactory loss. Am J Rhinol. 2004 Jan-Feb;18(1):29-33.

22 Stenner M, Vent J, Hüttenbrink KB, Hummel T, Damm M. Topical therapy in anosmia: relevance of steroid-responsiveness. Laryngoscope. 2008 Sep;118(9):1681-6.

23 Schriever VA, Merkonidis C, Gupta N, Hummel C, Hummel T. Treatment of smell loss with systemic methylprednisolone. Rhinology. 2012 Sep;50(3):284-9.

24 Kim DH, Kim SW, Hwang SH, Kim BG, Kang JM, Cho JH, et al. Prognosis of olfactory dysfunction according to etiology and timing of treatment. Otolaryngol Head Neck Surg. 2017 Feb;156(2):371-7.

25 Lam K, Tan BK, Lavin JM, Meen E, Conley DB. Comparison of nasal sprays and irrigations in the delivery of topical agents to the olfactory mucosa. Laryngoscope. 2013 Dec; 123(12):2950-7.
26 Shu CH, Lee PL, Shiao AS, Chen KT, Lan MY. Topical corticosteroids applied with a squirt system are more effective than a nasal spray for steroid-dependent olfactory impairment. Laryngoscope. 2012 Apr;122(4):747-50.

27 Mori E, Merkonidis C, Cuevas M, Gudziol V, Matsuwaki Y, Hummel T. The administration of nasal drops in the "Kaiteki" position allows for delivery of the drug to the olfactory cleft: a pilot study in healthy subjects. Eur Arch Otorhinolaryngol. 2016 Apr;273(4):939-43.

28 Kanemitsu Y, Kurokawa R, Ono J, Fukumitsu K, Takeda N, Fukuda S, et al. Increased serum periostin levels and eosinophils in nasal polyps are associated with the preventive effect of endoscopic sinus surgery for asthma exacerbations in chronic rhinosinusitis patients. Int Arch Allergy Immunol. 2020;181(11):86270.

29 Guilemany JM, Mariño-Sánchez FS, Angrill J, Alobid I, Centellas S, Pujols L, et al. The importance of smell in patients with bronchiectasis. Respir Med. 2011 Jan;105(1):44-9.

30 Seiden AM. Postviral olfactory loss. Otolaryngol Clin North Am. 2004 Dec;37:1159-66.

31 Jafek BW, Murrow B, Michaels R, Restrepo D, Linschoten M. Biopsies of human olfactory epithelium. Chem Senses. 2002 Sep;27(7): 623-8.

32 Nishimura T, Teranishi S, Kawashima A Ishimaru T, Miwa T, Furukawa M. Glucocorticoid enhances $\mathrm{Na}(+) / \mathrm{K}(+)$ ATPase mRNA expression in rat olfactory mucosa during regeneration: a possible mechanism for recovery from olfactory disturbance. Chem Senses. 2002 Jan;27(1):13-21.

33 Takanosawa M, Nishino H, Ohta Y, Ichimura K. Glucocorticoids enhance regeneration of murine olfactory epithelium. Acta Otolaryngol. 2009 Sep;129(9):1002-9.

34 Choi R, Goldstein B. Olfactory epithelium: cells, clinical disorders, and insights from an adult stemm cell niche. Laryngoscope Investig Otolaryngol. 2018 Feb;3(1):35-42.

35 Haehner A, Draf J, Dräger S, de With K, Hummel T. Predictive value of sudden olfactory loss in the diagnosis of COVID-19. ORL J Otorhinolaryngol Relat Spec. 2020;82(4): 175-80.

36 WHO. Corticosterids for COVID-19: Living guidance. 2020 Sep 2. Available from: https:// www.who.int/publications-detail-redirect/ WHO-2019-nCoV-Corticosteroids-2020.1 Assessed 2020 Sep 14.

37 Addison AB, Wong B, Ahmed T, Macchi A Konstantinidis I, Huart C, et al. Clinical Olfactory Working Group consensus statement on the treatment of postinfectious olfactory dysfunction. J Allergy Clin Immunol. 2021 Jan 13;S0091-6749(21):00004-X. 